\title{
On the higher Whitehead product
}

\author{
Marek Golasiński ${ }^{1}$ - Thiago de Melo²
}

Received: 1 May 2015 / Accepted: 6 November 2015 / Published online: 15 October 2016

(C) The Author(s) 2016. This article is published with open access at Springerlink.com

\begin{abstract}
Porter's approach is used to derive some properties of higher order Whitehead products, similar to those ones for triple products obtained by Hardie. Computations concerning the higher order Whitehead product for spheres and projective spaces are presented as well.
\end{abstract}

Keywords Generalized separation element - Generalized Whitehead product . $G$-space $\cdot$ Higher order Whitehead product

Mathematics Subject Classification Primary 55Q15; Secondary 55Q35

Dedicated to Ronnie Brown on the occasion of his eightieth birthday.

Communicated by Tim Porter and George Janelidze.

Thiago de Melo was supported by FAPESP 2014/21926-1.

$凶$ Marek Golasiński

marekg@matman.uwm.edu.pl

Thiago de Melo

tmelo@rc.unesp.br

1 Faculty of Mathematics and Computer Science, University of Warmia and Mazury, Słoneczna 54 Street, 10-710 Olsztyn, Poland

2 Instituto de Geociências e Ciências Exatas, UNESP-Univ Estadual Paulista, Av. 24A, 1515, Bela Vista, Rio Claro, SP CEP 13.506-900, Brazil 


\section{Introduction}

Whitehead products play an important role in algebraic topology and its applications. The classical Whitehead product $[f, g]$ of (homotopy classes of) maps $f: \mathbb{S}^{m} \rightarrow X$ and $g: \mathbb{S}^{n} \rightarrow X$ is (the homotopy class) represented by a map $h: \mathbb{S}^{m+n-1} \rightarrow X$ and defined by means of the so called Whitehead map $\omega: \mathbb{S}^{m+n-1} \rightarrow \mathbb{S}^{m} \vee \mathbb{S}^{n}$, the attaching map for the $(m+n)$-cell of $\mathbb{S}^{m} \times \mathbb{S}^{n}$.

Arkowitz [2] constructed a generalization $\omega: \Sigma(A \wedge B) \rightarrow \Sigma A \vee \Sigma B$ of the Whitehead map to define the generalized Whitehead product $[f, g]$ of maps $f: \Sigma A \rightarrow$ $X$ and $g: \Sigma B \rightarrow X$. Then Zeeman [13] and Hardie [8] generalized the Whitehead product in a different context, that is, they defined a triple spherical Whitehead product $\left[f_{1}, f_{2}, f_{3}\right]$ of maps $f_{i}: \mathbb{S}^{m_{i}} \rightarrow X$ for $i=1,2,3$. The main result from [8] deals with the $r$ th order spherical Whitehead product $\left[f_{1}, \ldots, f_{r}\right]$ for maps $f_{i}: \mathbb{S}^{m_{i}} \rightarrow X$ with $r \geq 3$ defined in [9], and in particular, it states that the triple product $\left[f_{1}, f_{2}, f_{3}\right]$ is a coset of a subgroup of $\pi_{m-1}(X)$, where $m=m_{1}+m_{2}+m_{3}$. Many properties which hold for the classical Whitehead product still hold for the triple one as well.

Porter's approach [15] was to construct the Whitehead map $\omega_{r}$ for more than two spaces (see Eq. (5)). Hardie's construction from [9] was generalized in [15], where the $r$ th order generalized Whitehead product of maps $f_{i}: \Sigma A_{i} \rightarrow X$ for $i=1, \ldots, r$, with $r \geq 2$ was introduced. Then the 2 nd order Whitehead product coincides with the generalized Whitehead product studied by Arkowitz [2].

Higher order Whitehead products are secondary, tertiary, etc. analogues of ordinary Whitehead products. They first appeared in the late 1960's as a part of a research theme studying higher products, or simply higher structures, in homotopy theory. They are vital for understanding the homotopy theory of certain basic objects, such as an iterated product of spaces, and their maps into other spaces. Recently, higher Whitehead products have re-emerged as key players in the homotopy theory of polyhedral products. These are important objects in toric topology and are being increasingly used in geometric group theory and graph theory. Porter's construction is very useful in many mathematical constructions. For example, given a simplicial complex $K$ on $n$ vertices, Davis and Januszkiewicz [6] associated two fundamental objects of toric topology: the moment-angle complex $\mathcal{Z}_{K}$ and the Davis-Januszkiewicz space $D J_{K}$. The homotopy fibration sequence

$$
\mathcal{Z}_{K} \stackrel{\tilde{\omega}}{\rightarrow} D J_{K} \rightarrow \prod_{i=1}^{n} \mathbb{C} P^{\infty}
$$

and its generalization have been studied in [7,11], respectively to show that $\tilde{\omega}: \mathcal{Z}_{K} \rightarrow$ $D J_{K}$ is a sum of higher and iterated Whitehead products for appropriate complexes $K$.

Next, let $\mathcal{F}\left(\mathbb{R}^{n+1}, m\right)$ be the Euclidean ordered configuration space. By Salvatore [17, Theorem 7], the homotopy type of $\mathcal{F}\left(\mathbb{R}^{n+1}, m\right)$ for $n \geq 2$ admits a minimal cellular model

$$
*=X_{0} \subseteq X_{n} \subseteq X_{2 n} \subseteq \cdots \subseteq X_{m n}
$$

whose cells are attached via higher order Whitehead products. 
In this paper, we work with triple and $r$ th order Whitehead products. The aim of Sect. 1 is to fix some notations, recall definitions and necessary results from $[1,2]$ and present properties on separation elements, and the relative generalized Whitehead product as well. Section 2 expounds the main facts from [15] on $r$ th Whitehead products which are used to discuss the main result from [8]. In Sect. 3, based on some relations from [20, (5.5), (5.8) and Proposition 5.11], we prove Proposition 4, the main result of that section, which deals with the non-triviality problem of the triple product $\left[\eta_{4}, \eta_{4}^{2}, 2 \iota_{4}\right] \subseteq \pi_{14}\left(\mathbb{S}^{4}\right)$, stated by Hardie [8, Section 5]. The main result of Sect. 4 is Theorem 4 which extends the result [8, Theorem 4.3] to higher order Whitehead products. Finally, Sect. 5 is devoted to some computations concerning the $r$ th order Whitehead product for spheres and projective spaces. In particular, Proposition 5 shows that there are spaces $X$ such that the $r$ th order generalized Whitehead product $\left[0_{1}, \ldots, 0_{r}\right]$ contains a non-trivial element provided $0_{i}: \mathbb{S}^{m_{i}} \rightarrow X$ are trivial for $i=1, \ldots, r$ with $r \geq 4$.

\section{Preliminaries}

Denote by $\pi(X, Y)$ the set of (based) homotopy classes of (based) maps $X \rightarrow Y$. In the sequel we do not distinguish between a map and its homotopy class. Write $C(-)$ and $\Sigma(-)$ for the reduced cone and the reduced suspension functors, respectively. As in Toda [20, p. 16], $\iota_{n}: \mathbb{S}^{n} \rightarrow \mathbb{S}^{n}$ denotes the identity map of the $n$-sphere $\mathbb{S}^{n}$, and $\eta_{n}=\Sigma^{n-2} \eta_{2}: \mathbb{S}^{n+1} \rightarrow \mathbb{S}^{n}$ for $n \geq 2$, is the iterated suspension of the Hopf map $\eta_{2}: \mathbb{S}^{3} \rightarrow \mathbb{S}^{2}$. We also use freely other symbols from [20, pp. 39-50 and pp. 172-185] and write $\iota_{X}$ for the identity map on a space $X$.

\subsection{Relative generalized Whitehead product}

Let $A$ and $B$ be spaces. Given maps $f: \Sigma A \rightarrow X$ and $g: \Sigma B \rightarrow X$, Arkowitz constructed in [2] the Whitehead map

$$
\omega: \Sigma(A \wedge B) \rightarrow \Sigma A \vee \Sigma B
$$

to define the generalized Whitehead product $[f, g]$ as the map $\nabla(f \vee g) \omega: \Sigma(A \wedge$ $B) \rightarrow X$, where $\nabla: X \vee X \rightarrow X$ is the folding map and $A \wedge B$ is the smash product. Also, from [2, Proposition 3.3], the product is anti-commutative, that is, $[g, f]=-(\Sigma \sigma)^{*}[f, g]$, where $\sigma: B \wedge A \rightarrow A \wedge B$ is the homeomorphism determined by the map $B \times A \rightarrow A \times B$.

In [1], Ando defined the relative generalized Whitehead product, briefly described as follows. Let $i_{Z}: Z \hookrightarrow C Z$ be the inclusion map. Given $k: X \rightarrow Y$, we say that $\left(h_{1}, h_{2}\right): i_{Z} \rightarrow k$ is a pair-map provided the diagram

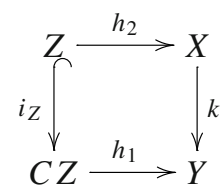


is commutative. Write $\pi_{1}(Z, k)$ for the set of homotopy classes of pair-maps $\left(h_{1}, h_{2}\right)$ : $i_{Z} \rightarrow k$.

Note that if $Z=\Sigma Z^{\prime}$ then the standard co- $H$-structure $v: \Sigma Z^{\prime} \rightarrow \Sigma Z^{\prime} \vee \Sigma Z^{\prime}$ leads to a pair-map

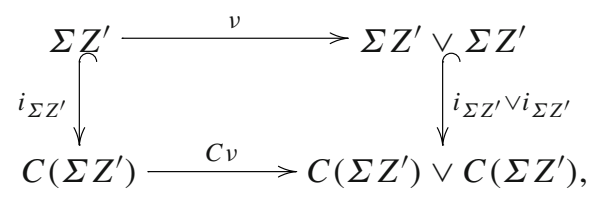

which determines a group structure on $\pi_{1}(Z, k)$. Moreover, this group is abelian provided $Z^{\prime}=\Sigma Z^{\prime \prime}$. In particular, if $k: X_{0} \hookrightarrow X$ is an inclusion map and $Z=\mathbb{S}^{n}$, we obtain the ordinary relative homotopy groups $\pi_{1}\left(\mathbb{S}^{n}, k\right)=\pi_{n+1}\left(X, X_{0}\right)=$ $\left[\left(\mathbb{D}^{n+1}, \mathbb{S}^{n}\right),\left(X, X_{0}\right)\right]$, where $\mathbb{D}^{n+1}=C \mathbb{S}^{n}$ is the $(n+1)$-disc.

Further, the map $k: X \rightarrow Y$ implies the dual Puppe long exact sequence

$$
\cdots \rightarrow \pi\left(\Sigma^{2} Z, X\right) \stackrel{k_{*}}{\rightarrow} \pi\left(\Sigma^{2} Z, Y\right) \stackrel{j_{*}}{\rightarrow} \pi_{1}(\Sigma Z, k) \stackrel{\delta_{*}}{\rightarrow} \pi(\Sigma Z, X) \stackrel{k_{*}}{\rightarrow} \cdots
$$

For $k=i_{\Sigma A} \vee \iota_{\Sigma B}$, according to [1, Section 2(b)], there is a commutative diagram

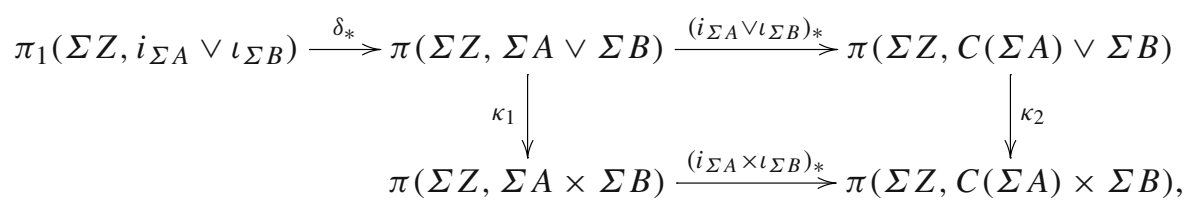

where the vertical maps $\kappa_{i}$ for $i=1,2$ are induced by the inclusions of the wedge into the product, and the top row is exact.

By [15, Theorem (2.3)], $\left(i_{\Sigma A} \times \iota_{\Sigma B}\right)_{*} \kappa_{1}(\omega)=0$ and by the commutativity of the diagram above, and the fact that $\kappa_{2}$ is isomorphism, $\left(i_{\Sigma A} \vee \iota_{\Sigma B}\right)_{*}(\omega)=0$. Note that $\left(i_{\Sigma A} \vee \iota_{\Sigma B}\right)_{*}: \pi(\Sigma Z, \Sigma A \vee \Sigma B) \rightarrow \pi(\Sigma Z, C(\Sigma A) \vee \Sigma B)$ is an epimorphism, and so $\delta_{*}$ is a monomorphism. Thus, by the exactness of the top row, there is a unique element $\bar{\omega} \in \pi_{1}\left(\Sigma Z, i_{\Sigma A} \vee \iota_{\Sigma B}\right)$ such that $\delta_{*}(\bar{\omega})=\omega$.

Let $k: X \rightarrow Y$ and $Z=A \wedge B$ in the above. Then, given $f=\left(f_{1}, f_{2}\right) \in \pi_{1}(\Sigma A, k)$ and $g \in \pi(\Sigma B, X)$, the element $\bar{\omega}$ determines a pair-map $\left(\omega^{\prime}, \omega\right)$ commuting the diagram

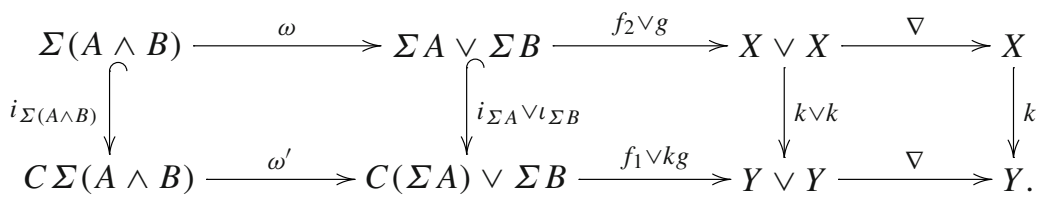


As in [1], the relative generalized Whitehead product $[f, g]_{R} \in \pi_{1}(\Sigma(A \wedge B), k)$ is defined by the pair-map

$$
\left(\nabla\left(f_{1} \vee k g\right), \nabla\left(f_{2} \vee g\right)\right)_{*}(\bar{\omega})=\left(\nabla\left(f_{1} \vee k g\right) \omega^{\prime}, \nabla\left(f_{2} \vee g\right) \omega\right)
$$

Also therein, Ando compares his construction to the one introduced by Hardie in [8]. We describe that here to obtain Proposition 2.

First, since the generalized Whitehead product $[0, g]$ is trivial and the product $\Sigma A \times \Sigma B$ is a push-out, we obtain a map $\lambda_{A}(g): C \Sigma(A \wedge B) \rightarrow X$ commuting the diagram

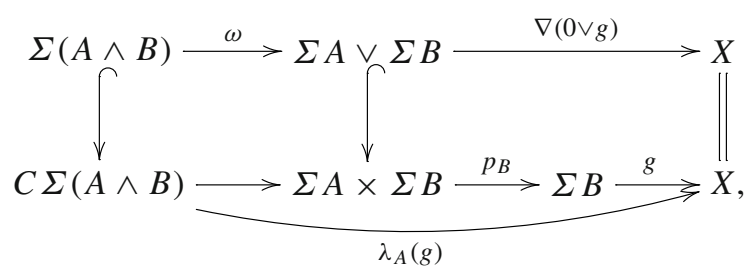

where $p_{B}: \Sigma A \times \Sigma B \rightarrow \Sigma B$ is the projection map.

Let $\left[f_{1}, k g\right]^{\prime}: C \Sigma(A \wedge B) \rightarrow Y$ be given by

$$
\left[f_{1}, k g\right]^{\prime}[y, t]= \begin{cases}\lambda_{A}(k g)[y, 2 t], & 0 \leq t \leq \frac{1}{2}, \\ {\left[f_{1} \sigma_{2 t-1}^{\prime}, k g\right](y),} & \frac{1}{2} \leq t \leq 1,\end{cases}
$$

where $y \in \Sigma(A \wedge B)$, and $\sigma_{2 t-1}^{\prime}: \Sigma A \rightarrow C \Sigma A$ is given by $\sigma_{2 t-1}^{\prime}(x)=[x, 2 t-1]$ for $x \in \Sigma A$. Thus, by [1, Theorem (4.6)] we obtain

$$
[f, g]_{R}=\left(\left[f_{1}, k g\right]^{\prime},\left[f_{2}, g\right]\right) .
$$

Further,

$$
\varphi\left[f_{1}, k g\right]^{\prime}=\left[\varphi f_{1}, \varphi k g\right]^{\prime}
$$

for $\varphi: Y \rightarrow Z$ and

$$
\left[(C \Sigma \alpha)^{*} f_{1},(\Sigma \beta)^{*} k g\right]^{\prime}=(C \Sigma(\alpha \wedge \beta))^{*}\left[f_{1}, k g\right]^{\prime}
$$

for $\alpha: A^{\prime} \rightarrow A$ and $\beta: B^{\prime} \rightarrow B$.

Next, suppose that $A=\Sigma A^{\prime}$ and consider $\left[f_{2}, g\right]^{\prime \prime}: \Sigma(A \wedge B) \rightarrow X$ given by

$$
\left[f_{2}, g\right]^{\prime \prime}[y, t]= \begin{cases}\lambda_{A}(g)[y, 4 t], & 0 \leq t \leq \frac{1}{4}, \\ {\left[f_{2} \sigma_{\frac{1}{2}(4 t-1)}, g\right](y),} & \frac{1}{4} \leq t \leq \frac{3}{4} \\ \lambda_{A}(g)[y, 4-4 t], & \frac{3}{4} \leq t \leq 1\end{cases}
$$


where $y \in \Sigma(A \wedge B)$, and $\sigma_{\frac{1}{2}(4 t-1)}: A \rightarrow \Sigma A$ is given by $\sigma_{\frac{1}{2}(4 t-1)}(a)=\left[a, \frac{1}{2}(4 t-\right.$ $1)]$ for $a \in A$. Note that for $t=\frac{1}{4}, f_{2} \sigma_{0}=0$ and $\lambda_{A}(g)[y, 1]=[0, g](y)$, and the same for $t=\frac{3}{4}$. So, the map $\left[f_{2}, g\right]^{\prime \prime}$ is well defined and $\left[f_{2}, g\right]^{\prime \prime}=\left[f_{2}, g\right]$ (cf. $[8$, Theorem (2.4)]).

Remark 1 We work with relative Whitehead products for an inclusion map $k: X_{0} \hookrightarrow$ $X$. It means that (homotopy classes of) maps $f:(C \Sigma A, \Sigma A) \rightarrow\left(X, X_{0}\right)$ and $g: \Sigma B \rightarrow X_{0}$ determine the map of pairs

$$
[f, g]_{R}:(C \Sigma(A \wedge B), \Sigma(A \wedge B)) \rightarrow\left(X, X_{0}\right) .
$$

We finish this section with some properties of the relative product $[-,-]_{R}$.

Let $f, f_{i} \in \pi_{1}(\Sigma A, k)$ and $g, g_{i} \in \pi(\Sigma B, X)$ for $i=1,2$. According to [1, Proposition (4.5)], if $A$ and $B$ are suspensions then $\left[f, g_{1}+g_{2}\right]_{R}=\left[f, g_{1}\right]_{R}+\left[f, g_{2}\right]_{R}$ and $\left[f_{1}+f_{2}, g\right]_{R}=\left[f_{1}, g\right]_{R}+\left[f_{2}, g\right]_{R}$. Also, $\left[1\right.$, Theorem (4.8)] states that $[g, f]_{R}=$ $-(C \Sigma \sigma, \Sigma \sigma)^{*}[f, g]_{R}$, where $\sigma: B \wedge A \rightarrow A \wedge B$ is the canonical homeomorphism.

\subsection{Generalized separation element}

Given maps $f, g: C A \rightarrow X$ such that $f_{\mid A}=g_{\mid A}$, following James [12, Section 10] and Tsuchida [21, Section 3], we define the generalized separation element as (the homotopy class of ) the map $d(f, g): \Sigma A \rightarrow X$ given by:

$$
d(f, g)[a, t]= \begin{cases}f[a, 2 t], & 0 \leq t \leq \frac{1}{2} \\ g[a, 2-2 t], & \frac{1}{2} \leq t \leq 1\end{cases}
$$

Further, if $A=\Sigma A^{\prime}$ then we consider

$$
f+g: C \Sigma A^{\prime} \stackrel{C \nu}{\longrightarrow} C\left(\Sigma A^{\prime}\right) \vee C\left(\Sigma A^{\prime}\right) \stackrel{\nabla(f \vee g)}{\longrightarrow} X .
$$

Then, following (mutatis mutandis) Hardie [8, Section 1] and James [12, Section 10], we may state:

Proposition 1 Let $f, g, h: C A \rightarrow X$ be maps such that $f_{\mid A}=g_{\mid A}=h_{\mid A}$, and let $k: X \rightarrow Y$ be any map. Then:

(i) triviality: $d(f, f)=0$;

(ii) triangularity: $d(f, g)+d(g, h)=d(f, h)$;

(iii) antisymmetry: $d(g, f)=-d(f, g)$;

(iv) naturality: $d(k f, k g)=k d(f, g)$;

(v) co-naturality: $d\left((C \alpha)^{*} f,(C \alpha)^{*} g\right)=(\Sigma \alpha)^{*} d(f, g)$ for $\alpha: A^{\prime} \rightarrow A$.

Moreover, let $f_{i}, g_{i}: C \Sigma A \rightarrow X$ be maps for $i=1,2$ such that $f_{1 \mid \Sigma A}=f_{2 \mid \Sigma A}$ and $g_{1 \mid \Sigma A}=g_{2 \mid \Sigma A}$. Then:

(vi) $d\left(f_{1}+g_{1}, f_{2}+g_{2}\right)=d\left(f_{1}, f_{2}\right)+d\left(g_{1}, g_{2}\right)$; 
(vii) if $\varphi: \Sigma A \rightarrow X$ then there exists $f^{\prime}: C A \rightarrow X$ such that $f_{\mid A}^{\prime}=f_{\mid A}$ and $-\varphi=d\left(f, f^{\prime}\right)$

Let $j_{*}$ and $\delta_{*}$ be the maps as in the exact sequence (2). Then:

(viii) if $\delta_{*}\left(f^{\prime}, f^{\prime \prime}\right)=0$ for some $\left(f^{\prime}, f^{\prime \prime}\right) \in \pi_{1}(\Sigma A, k)$ and $g \in \pi(C \Sigma A, X)$ such that $g_{\mid \Sigma A}=f_{\mid \Sigma A}^{\prime}$ then $j_{*} d\left(f^{\prime}, k g\right)=\left(f^{\prime}, f^{\prime \prime}\right)$. In addition for any $h \in j_{*}^{-1}\left(f^{\prime}, f^{\prime \prime}\right)$ there exists $g^{\prime} \in \pi(C \Sigma A, X)$ with $g_{\mid \Sigma A}^{\prime}=f_{\mid \Sigma A}^{\prime}$ such that $h=d\left(f^{\prime}, k^{\prime}\right)$.

Proof Since the items (i)-(vi) follow directly from the definition of $d$, we just prove (vii) and sketch a proof of (viii).

For (vii), let $q: A \times I \rightarrow C A$ be the quotient map such that the inclusion $A \hookrightarrow C A$ is given by $a \mapsto q(a, 1)=[a, 1]$ for $a \in A$. Define $f^{\prime}: C A \rightarrow X$ by

$$
f^{\prime}[a, t]= \begin{cases}\varphi[a, 2 t], & 0 \leq t \leq \frac{1}{2}, \\ f q(a, 2 t-1), & \frac{1}{2} \leq t \leq 1\end{cases}
$$

and note that for $t=\frac{1}{2}, \varphi[a, 1]=*=f q(a, 0)$, and for $t=1, f^{\prime}[a, 1]=f q(a, 1)=$ $f[a, 1]$, that is, $f_{\mid A}=f_{\mid A}^{\prime}$. After some computations and changes of parameters we can show that:

$$
d\left(f, f^{\prime}\right)[a, t]= \begin{cases}f\left[a, \frac{8}{3} t\right], & 0 \leq t \leq \frac{3}{8} \\ f\left[a, 2-\frac{8}{3} t\right], & \frac{3}{8} \leq t \leq \frac{3}{4} \\ \varphi[a, 4(1-t)], & \frac{3}{4} \leq t \leq 1\end{cases}
$$

Since the first two parts provide a map homotopic to $d(f, f)=0$ and the third part is homotopic to $-\varphi$, we obtain $-\varphi=d\left(f, f^{\prime}\right)$.

For (viii), we note that the map $j_{*}: \pi\left(\Sigma^{2} A, Y\right) \rightarrow \pi_{1}(\Sigma A, k)$ is determined by the map $(C \Sigma A, \Sigma A) \rightarrow\left(\Sigma^{2} A, *\right)$. Then, we simply mimic the proof of [8, Theorem 1.9].

We finish this section by stating a relation between the separation element and the products defined in Sect. 1.1 (cf. [8, (2.7)]).

Proposition 2 Let $f=\left(f_{1}, f_{2}\right), f^{\prime}=\left(f_{1}^{\prime}, f_{2}^{\prime}\right) \in \pi_{1}(\Sigma A, k)$ and $g \in \pi(\Sigma B, X)$, where $k: X \rightarrow Y$. Suppose that $f_{2}=f_{2}^{\prime}: \Sigma A \rightarrow X$. Then

$$
d\left(\left[f_{1}, k g\right]^{\prime},\left[f_{1}^{\prime}, k g\right]^{\prime}\right)=\left[d\left(f_{1}, f_{1}^{\prime}\right), k g\right]: \Sigma^{2}(A \wedge B) \rightarrow Y .
$$

Proof Just observe that the restrictions of $\left[f_{1}, k g\right]^{\prime}$ and $\left[f_{1}^{\prime}, k g\right]^{\prime}$ to $\Sigma(A \wedge B)$ are equal to $k_{*}\left[f_{2}, g\right]$ and $k_{*}\left[f_{2}^{\prime}, g\right]$, respectively. Since $f_{2}=f_{2}^{\prime}$, we get $k_{*}\left[f_{2}, g\right]=k_{*}\left[f_{2}^{\prime}, g\right]$ and $d\left(\left[f_{1}, k g\right]^{\prime},\left[f_{1}^{\prime}, k g\right]^{\prime}\right)$ is defined. Then by the definition of the generalized separation element and the discussion above

$$
d\left(\left[f_{1}, k g\right]^{\prime},\left[f_{1}^{\prime}, k g\right]^{\prime}\right)=\left[d\left(f_{1}, f_{1}^{\prime}\right), k g\right]^{\prime \prime}=\left[d\left(f_{1}, f_{1}^{\prime}\right), k g\right]
$$

and the proof follows. 


\section{Higher order generalized Whitehead product}

Let $r \geq 2$ be an integer and denote by $\underline{A}=\left(A_{1}, \ldots, A_{r}\right)$ an $r$-tuple of topological spaces with base points $*$. The fat wedge of $\underline{A}$ is the space

$$
F W(\underline{A})=\left\{\left(a_{1}, \ldots, a_{r}\right) \in A_{1} \times \cdots \times A_{r} \mid \text { at least one } a_{i}=*\right\} .
$$

If $r=2$, then $F W(\underline{A})=A_{1} \vee A_{2}$ is the wedge sum.

Following Porter's notation [15], let $T_{0}(\underline{A})=A_{1} \times \cdots \times A_{r}$ and $T_{S}(\underline{A}) \subseteq T_{0}(\underline{A})$ be the subset of points $\left(a_{1}, \ldots, a_{r}\right)$ with at least $s$ coordinates $a_{i}=*$ for $s=1, \ldots, r$. Thus, $T_{r-1}(\underline{A})=A_{1} \vee \cdots \vee A_{r}$ is the wedge sum and $T_{1}(\underline{A})=F W(\underline{A})$ is the fat wedge. Also, we write $\Lambda(\underline{A})=A_{1} \wedge \cdots \wedge A_{r}$ and $\Sigma(\underline{A})=\left(\Sigma A_{1}, \ldots, \Sigma A_{r}\right)$.

Remark 2 In the sequel, we need to take some coordinate $a_{i_{0}}=*$. To do this, we define

$$
T_{0}^{(i)}(\underline{A})=\prod_{\substack{j=1 \\ j \neq i}}^{r} A_{j}, \quad \Lambda^{(i)}(\underline{A})=\bigwedge_{\substack{j=1 \\ j \neq i}}^{r} A_{j}
$$

for $i=1, \ldots, r$. In this way, the subset $T_{s}^{(i)}(\underline{A}) \subseteq T_{0}^{(i)}(\underline{A})$ is defined for $s=$ $1, \ldots, r-1$ and has a self-explanatory notation. Also, there are canonical embeddings $\Psi_{s}^{(i)}: T_{s}^{(i)}(\underline{A}) \hookrightarrow T_{s+1}(\underline{A})$ for any $s=0, \ldots, r-1$.

In [15], Porter constructed the generalized Whitehead map (cf. (1))

$$
\omega_{r}: \Sigma^{r-1} \Lambda(\underline{A}) \rightarrow T_{1} \Sigma(\underline{A})
$$

to define the $r$ th order generalized Whitehead product $\omega_{r}(f) \in \pi\left(\Sigma^{r-1} \Lambda(\underline{A}), X\right)$ of a map $f: T_{1} \Sigma(\underline{A}) \rightarrow X$ as the composite

$$
\omega_{r}(f): \Sigma^{r-1} \Lambda(\underline{A}) \stackrel{\omega_{r}}{\longrightarrow} T_{1} \Sigma(\underline{A}) \stackrel{f}{\longrightarrow} X .
$$

Given $f_{i}: \Sigma A_{i} \rightarrow X$ for $i=1, \ldots, r$, let $f_{1} \vee \cdots \vee f_{r}: T_{r-1} \Sigma(\underline{A}) \rightarrow X$ be the wedge sum map. The (possibly empty) set

$$
\left[f_{1}, \ldots, f_{r}\right]=\left\{\omega_{r}(f) \in \pi\left(\Sigma^{r-1} \Lambda(\underline{A}), X\right)\right\}
$$

for all $f: T_{1} \Sigma(\underline{A}) \rightarrow X$ extending $f_{1} \vee \cdots \vee f_{r}$, is called the $r$ th order generalized Whitehead product of $f_{1}, \ldots, f_{r}$. Hardie [9] gave the definition of $\left[f_{1}, \ldots, f_{r}\right]$ when all the $A_{i}$ 's are spheres (called the $r$ th order spherical Whitehead product).

In virtue of [15, Theorem (2.7)], it is non-empty if and only if all the lower products $\left[f_{m_{1}}, \ldots, f_{m_{k}}\right]$ for $1 \leq m_{1} \leq \cdots \leq m_{k} \leq r$ with $k=2, \ldots, r-1$ contain the zero element 0 . We note that the set $\left[f_{1}, \ldots, f_{r}\right]$ can be empty even if $f_{i}=0$ for some $i$. This is the case for $\left[0, \iota_{2}, \iota_{2}\right]$, since the classical Whitehead product $\left[\iota_{2}, \iota_{2}\right]=2 \eta_{2} \neq 0$, by [20, p. 39]. 
Remark 3 In the sequel we will use the same notation $\sigma$ for a permutation of the set $\{1, \ldots, r\}$ and for its induced homeomorphism $A_{1} \wedge \cdots \wedge A_{r} \rightarrow A_{\sigma(1)} \wedge \cdots \wedge A_{\sigma(r)}$.

Proposition 3 Let $f_{i}: \Sigma A_{i} \rightarrow X$ for $i=1, \ldots, r$ and $\sigma \in S_{r}$ be a permutation of the set $\{1, \ldots, r\}$. Then

$$
\left[f_{\sigma(1)}, \ldots, f_{\sigma(r)}\right]=\operatorname{sgn}(\sigma)\left(\Sigma^{r-1} \sigma\right)^{*}\left[f_{1}, \ldots, f_{r}\right] .
$$

Proof Denote by $\sigma \underline{A}=\left(A_{\sigma(1)}, \ldots, A_{\sigma(r)}\right)$ the image of $\underline{A}$ under the permutation $\sigma \in S_{r}$. It is clear that there is a commutative diagram

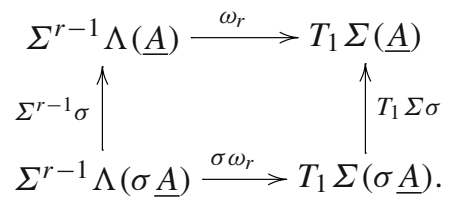

Let $f_{\sigma(1)} \vee \cdots \vee f_{\sigma(r)}=\left(T_{r-1} \Sigma \sigma\right)^{*}\left(f_{1} \vee \cdots \vee f_{r}\right): T_{r-1} \Sigma(\sigma \underline{A}) \rightarrow X$. So, if $f: T_{1} \Sigma(\underline{A}) \rightarrow X$ is an extension of $f_{1} \vee \cdots \vee f_{r}$ then $\sigma f=\left(T_{1} \Sigma \sigma\right)^{*}(f)$ : $T_{1} \Sigma(\sigma \underline{A}) \rightarrow X$ is an extension of $f_{\sigma(1)} \vee \cdots \vee f_{\sigma(r)}$. Finally, note that $\sigma \omega_{r}(\sigma f)=$ $\operatorname{sgn}(\sigma)\left(\Sigma^{r-1} \sigma\right)^{*}\left(\omega_{r}(f)\right)$ and the proof follows.

According to [15, Theorem (2.3)] there is a homotopy equivalence

$$
T_{1} \Sigma(\underline{A}) \cup_{\omega_{r}} C\left(\Sigma^{r-1} \Lambda(\underline{A})\right) \stackrel{\simeq}{\rightarrow} T_{0} \Sigma(\underline{A}) .
$$

So, we have a pair-map $\left(\Omega_{r}, \omega_{r}\right)$ commuting the diagram

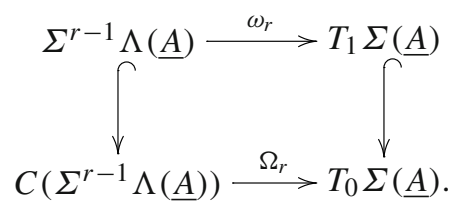

Considering the spaces and inclusions as in Remark 2, let

$$
\theta: \bigvee_{i=1}^{r} \Sigma^{r-2} \Lambda^{(i)}(\underline{A}) \rightarrow T_{2} \Sigma(\underline{A})
$$

be given by $\theta_{\mid \Sigma^{r-2} \Lambda^{(i)}(\underline{A})}=\Psi_{1}^{(i)} \omega_{r}^{(i)}$, where $\omega_{r}^{(i)}: \Sigma^{r-2} \Lambda^{(i)}(\underline{A}) \rightarrow T_{1}^{(i)} \Sigma(\underline{A})$ is the generalized Whitehead map. Then, as a particular case of [15, Theorem (2.6)], we have:

Theorem 1 The following spaces are homotopy equivalent:

(i) $T_{1} \Sigma(\underline{A})$;

(ii) $T_{2} \Sigma(\underline{A}) \cup_{\theta} C\left(\bigvee_{i=1}^{r} \Sigma^{r-2} \Lambda^{(i)}(\underline{A})\right)$; 
(iii) $T_{2} \Sigma(\underline{A}) \cup_{\theta} \bigvee_{i=1}^{r} C\left(\Sigma^{r-2} \Lambda^{(i)}(\underline{A})\right)$.

Now, we discuss some results on the $r$ th order generalized Whitehead product $\left[f_{1}, \ldots, f_{r}\right]$ of maps $f_{i}: \Sigma A_{i} \rightarrow X$ for $i=1, \ldots, r$. First, we recall [15, Theorems (2.1)(d), (2.4), (2.5) and Remark (4)] and present a corollary of [15, Theorem (2.3)].

Theorem 2 (i) (Naturality) Let $f_{i}: \Sigma A_{i} \rightarrow \Sigma B_{i}, g_{i}: \Sigma B_{i} \rightarrow X$ and $k: X \rightarrow Y$ be maps for $i=1, \ldots, r$. Then, $k_{*}\left[g_{1}, \ldots, g_{r}\right] \subseteq\left[k g_{1}, \ldots, k g_{r}\right]$.

(ii) If $X$ is an $H$-space then $\omega_{r}(f)=0$.

(iii) Let $f_{i}: \Sigma A_{i} \rightarrow X$ be maps for $i=1, \ldots, r$. Then $0 \in\left[f_{1}, \ldots, f_{r}\right]$ if and only if $f_{1} \vee \cdots \vee f_{r}: T_{r-1} \Sigma(\underline{A}) \rightarrow X$ has an extension to $T_{0} \Sigma(\underline{A})$. Further, $\Sigma \omega_{r}(f)=0$ for $f: T_{1} \Sigma(\underline{A}) \rightarrow X$ extending $f_{1} \vee \cdots \vee f_{r}$.

Corollary 1 If $\left[f_{1}, \ldots, f_{r}\right] \neq \varnothing$ and $f_{i_{0}}=0_{i_{0}}$ for some $1 \leq i_{0} \leq r$ then $0 \in$ $\left[f_{1}, \ldots, f_{r}\right]$.

Proof In virtue of Proposition 3, we can suppose that $i_{0}=1$. By hypothesis the map $0 \vee f_{2} \vee \cdots \vee f_{r}: T_{r-1} \Sigma(\underline{A}) \rightarrow X$ has an extension $F_{1}: T_{1} \Sigma(\underline{A}) \rightarrow X$. Define $F: T_{0} \Sigma(\underline{A}) \rightarrow X$ by $F\left(x_{1}, \ldots, x_{r}\right)=F_{1}\left(*, x_{2}, \ldots, x_{r}\right)$ for $\left(x_{1}, \ldots, x_{r}\right) \in T_{0} \Sigma(\underline{A})$. Then, $F$ is an extension of $0_{1} \vee f_{2} \vee \cdots \vee f_{r}$, and the result follows by Theorem 2(iii).

Recall from [19] that a space $X$ is called a $G$-space if for any map $f: \mathbb{S}^{n} \rightarrow X$ and $n \geq 1$ the map $\nabla\left(\iota_{X} \vee f\right): X \vee \mathbb{S}^{n} \rightarrow X$ extends to $F: X \times \mathbb{S}^{n} \rightarrow X$. According to [19, Theorem 3.2], any $r$ th order spherical Whitehead product of a $G$-space contains zero.

Now, let $\mathcal{G}(A, X) \subseteq \pi(A, X)$ be the set of (homotopy classes of) maps $f: A \rightarrow X$ such that the maps $\nabla\left(\iota_{X} \vee f\right): X \vee A \rightarrow X$ extend to $F: X \times A \rightarrow X$. A space $X$ is called a $G$-space with respect to the space $A$ if $\mathcal{G}(A, X)=\pi(A, X)$.

Lemma 1 If $X$ is a $G$-space with respect to the spaces $A_{1}, \ldots, A_{r}$ then any map $\iota_{X} \vee f_{1} \vee \cdots \vee f_{r}: X \vee A_{1} \vee \cdots \vee A_{r} \rightarrow X$ extends to $F: X \times A_{1} \times \cdots \times A_{r} \rightarrow X$.

Proof The proof is by induction on $r$. Let $r=2$ and $F_{i}: X \times A_{i} \rightarrow X$ extending $\iota_{X} \vee f_{i}: X \vee A_{i} \rightarrow X$ for $i=1,2$. Then $F_{2}\left(F_{1} \times \iota_{A_{2}}\right): X \times A_{1} \times A_{2} \rightarrow X$ extends $\iota_{X} \vee f_{1} \vee f_{2}: X \vee A_{1} \vee A_{2} \rightarrow X$.

Suppose that the statement is true for $r-1$, that is, there exists $F: X \times A_{1} \times \cdots \times$ $A_{r-1} \rightarrow X$ extending $\iota_{X} \vee f_{1} \vee \cdots \vee f_{r-1}$.

Finally, let $F_{r}: X \times A_{r} \rightarrow X$ extending $\iota_{X} \vee f_{r}: X \vee A_{r} \rightarrow X$ and then the composite $F_{r}\left(F \times \iota_{A_{r}}\right): X \times T_{0}(\underline{A}) \rightarrow X$ extends $\iota_{X} \vee f_{1} \vee \cdots \vee f_{r}$, which completes the proof.

Referring to Williams [22], we say that a space $X$ has property $P_{r}$ if for every $f_{i}: \Sigma A_{i} \rightarrow X$ with $i=1, \ldots, r$, we have $0 \in\left[f_{1}, \ldots, f_{r}\right]$. Certainly, in view of Theorem 2(ii), any $H$-space has not only property $P_{r}$ for all $r \geq 2$ but 0 is the only element of $\left[f_{1}, \ldots, f_{r}\right]$. (Williams [22]: We note at this point that it is unresolved conjecture as to whether $X$ has property $P_{r}$ implies that 0 is the only element of $\left[f_{1}, \ldots, f_{r}\right]$.)

Directly from Theorem 2(iii) and Lemma 1 it follows: 
Corollary 2 Every $G$-space $X$ with respect to any $\Sigma A_{1}, \ldots, \Sigma A_{r}$ has property $P_{r}$. In particular, if $f: \Sigma A \rightarrow X$ and $X$ is a $G$-space with respect to any $\Sigma A$ then $0 \in[f, \stackrel{\times}{.}, f]$ for any $r \geq 2$.

In general, the generalized Whitehead product is not additive. But, in the sequel, we need an addition operation defined in [15] for some particular functions. We say that maps $f, g: T_{1} \Sigma(\underline{A}) \rightarrow X$ are compatible off the $i$ th coordinate if $f \Psi_{0}^{(i)}=g \Psi_{0}^{(i)}$ : $T_{0}^{(i)} \Sigma(\underline{A}) \rightarrow X$. If $A_{i}=\Sigma A_{i}^{\prime}$ is a suspension one defines an addition $+^{(i)}$ by

$$
\begin{aligned}
& \left(f+{ }^{(i)} g\right)\left(x_{1}, \ldots,\left[t_{i},\left[u, a_{i}^{\prime}\right]\right], \ldots, x_{r}\right) \\
& \quad= \begin{cases}f\left(x_{1}, \ldots,\left[t_{i},\left[2 u, a_{i}^{\prime}\right]\right], \ldots, x_{r}\right), & 0 \leq u \leq \frac{1}{2}, \\
g\left(x_{1}, \ldots,\left[t_{i},\left[2 u-1, a_{i}^{\prime}\right]\right], \ldots, x_{r}\right), & \frac{1}{2} \leq u \leq 1 .\end{cases}
\end{aligned}
$$

Also, one defines $-^{(i)} f$ by

$$
\left(-^{(i)} f\right)\left(x_{1}, \ldots,\left[t_{i},\left[u, a_{i}^{\prime}\right]\right], \ldots, x_{r}\right)=f\left(x_{1}, \ldots,\left[t_{i},\left[1-u, a_{i}^{\prime}\right]\right], \ldots, x_{r}\right) .
$$

Then, in view of [15, Theorem (2.13)], we can state:

Theorem 3 (i) $\omega_{r}\left(f+{ }^{(i)} g\right)=\omega_{r}(f)+\omega_{r}(g)$;

(ii) $\omega_{r}\left({ }^{(i)} f\right)=-\omega_{r}(f)$.

From this, it follows:

Corollary 3 If $f_{i}: \Sigma A_{i} \rightarrow X$ for $i=1, \ldots, r$ then

$$
\left[f_{1}, \ldots, n f_{i}, \ldots, f_{r}\right] \subseteq n\left[f_{1}, \ldots, f_{i}, \ldots, f_{r}\right]
$$

for any integer $n$ and $i=1, \ldots, r$.

\section{Triple spherical Whitehead products}

Zeeman [13, Section 3.3] associated with a triple of elements $f \in \pi_{p}(X), g \in \pi_{q}(X)$ and $h \in \pi_{r}(X)(p, q, r \geq 2)$, whose Whitehead products taken in pairs all vanish, a certain subset $[f, g, h]^{\prime}$ of $\pi_{p+q+r-1}(X)$. Hardie [8] sharpened this construction defining the triple spherical Whitehead product $[f, g, h] \subseteq[f, g, h]^{\prime}$ and proving in $[8$, Theorem 0.1$]$ that $[f, g, h]$ is a coset of the subgroup

$$
J(f, g, h)=\left[\pi_{q+r}(X), f\right]+\left[\pi_{p+r}(X), g\right]+\left[\pi_{p+q}(X), h\right]
$$

of $\pi_{p+q+r-1}(X)$. Thus, a trivial triple spherical Whitehead product means $[f, g, h]=$ $J(f, g, h)$ or equivalently, $0 \in[f, g, h]$. Hardie stated in [8, Section 5]: We do not know of a case when the triple (spherical) product $[f, g, h]$ is non-trivial for a sphere, and for $X=\mathbb{S}^{4}$, the triple product $\left[\eta_{4}, \eta_{4}^{2}, 2 \iota_{4}\right] \subseteq \pi_{14}\left(\mathbb{S}^{4}\right)$ is possibly non-trivial. Proposition 4 below shows that it is non-empty and has order fifteen. In order to prove this, we need to check first that all lower products vanish. We keep below the standard notations from Toda's book [20, pp. 39-50 and pp. 172-185]. 
Lemma 2 Given $\eta_{4} \in \pi_{5}\left(\mathbb{S}^{4}\right), \eta_{4}^{2} \in \pi_{6}\left(\mathbb{S}^{4}\right)$, and $\iota_{4} \in \pi_{4}\left(\mathbb{S}^{4}\right)$, the following classical Whitehead products vanish:

$$
\left[\eta_{4}, 2 \iota_{4}\right]=0, \quad\left[\eta_{4}^{2}, 2 \iota_{4}\right]=0, \quad\left[\eta_{4}, \eta_{4}^{2}\right]=0 .
$$

Proof Certainly, $\left[\eta_{4}, 2 \iota_{4}\right]=\left[2 \eta_{4}, \iota_{4}\right]=0$ and $\left[\eta_{4}^{2}, \iota_{4}\right]=\left[2 \eta_{4}^{2}, \iota_{4}\right]=0$. Further, by [20, (5.9), p. 44], $\eta_{3} \circ v_{4}=v^{\prime} \circ \eta_{6}$, by [20, (5.5), p. 42], $4 v_{n}=\eta_{n}^{3}$ for $n \geq 5$, and by [20, Proposition 5.6, p. 42], $\Sigma v^{\prime}$ has order four.

$\operatorname{But}\left[\eta_{4}, \eta_{4}^{2}\right]=\eta_{4} \circ\left[\iota_{5}, \eta_{5}\right]=\eta_{4} \circ\left[\iota_{5}, \iota_{5}\right] \circ \eta_{9}$ and $\left[\iota_{5}, \iota_{5}\right]=v_{5} \circ \eta_{8}$, by $[20,(5.10), \mathrm{p}$. 44]. Thus $\left[\eta_{4}, \eta_{4}^{2}\right]=\left(\eta_{4} \circ v_{5}\right) \circ \eta_{8} \circ \eta_{9}=\Sigma v^{\prime} \circ \eta_{7} \circ \eta_{8} \circ \eta_{9}=\Sigma v^{\prime} \circ 4 v_{7}=4 \Sigma v^{\prime} \circ \eta_{7}=0$.

To prove the next proposition, we recall from [20, (13.1), p. 172 and Proposition 13.6, p. 179] that

$$
\pi_{11}\left(\mathbb{S}^{4}\right)=\mathbb{Z}_{3}\left\{\alpha_{2}(4)\right\} \oplus \mathbb{Z}_{5}\left\{\alpha_{1}(4)\right\}
$$

and for the 2-primary components, in view of [20, Propositions 5.9, p. 44 and 5.11, p. 46], we have:

$$
\pi_{9}^{4}=\mathbb{Z}_{2}\left\{v_{4} \circ \eta_{7}^{2}\right\} \oplus \mathbb{Z}_{2}\left\{\Sigma v^{\prime} \circ \eta_{7}^{2}\right\}, \quad \quad \pi_{10}^{4}=\mathbb{Z}_{8}\left\{v_{4}^{2}\right\}
$$

Moreover, from [18, Proposition IV.5], Serre's isomorphism for any odd p-primary components

$$
\pi_{i-1}\left(\mathbb{S}^{2 m-1} ; p\right) \oplus \pi_{i}\left(\mathbb{S}^{4 m-1} ; p\right) \simeq \pi_{i}\left(\mathbb{S}^{2 m} ; p\right)
$$

is given by $(f, g) \mapsto \Sigma f+\left[\iota_{2 m}, \iota_{2 m}\right] \circ g$.

Proposition 4 The groups $\left[\pi_{9}\left(\mathbb{S}^{4}\right), \eta_{4}^{2}\right]$ and $\left[\pi_{10}\left(\mathbb{S}^{4}\right), \eta_{4}\right]$ are trivial. In particular, $J\left(\eta_{4}, \eta_{4}^{2}, 2 \iota_{4}\right)=\left[\pi_{11}\left(\mathbb{S}^{4}\right), 2 \iota_{4}\right]$ and it is a subgroup of $\pi_{14}\left(\mathbb{S}^{4}\right)$ with order fifteen. In addition, the triple spherical Whitehead product

$$
\left[\eta_{4}, \eta_{4}^{2}, 2 \iota_{4}\right]=\left(4 x\left(\nu_{4} \circ \sigma^{\prime}\right)+2 y\left(\Sigma \varepsilon^{\prime}\right)\right)+J\left(\eta_{4}, \eta_{4}^{2}, 2 \iota_{4}\right)
$$

for some $x, y \in\{0,1\}$.

Proof First, recall that for $f \in \pi_{k}\left(\mathbb{S}^{m}\right)$ and $g \in \pi_{l}\left(\mathbb{S}^{m}\right)$ with relatively prime orders, the Whitehead product $[f, g]=0$. Hence, because the orders of $\eta_{4}$ and $\eta_{4}^{2}$ are two, we can restrict to $\pi_{9}^{4}$ and $\pi_{10}^{4}$ only.

For the first statement notice that

$$
\left[v_{4}^{2}, \eta_{4}\right]=\left[v_{4}, \iota_{4}\right] \circ \Sigma\left(v_{6} \wedge \eta_{3}\right)
$$

and

$$
\left[v_{4} \circ \eta_{7}^{2}, \eta_{4}^{2}\right]=\left[v_{4}, \iota_{4}\right] \circ \Sigma\left(\eta_{6}^{2} \wedge \eta_{3}^{2}\right) .
$$


Because $\left[v_{4}, \iota_{4}\right]= \pm 2 v_{4}^{2}$ ([3, Corollary (7.4)]), we derive that $\left[v_{4}^{2}, \eta_{4}\right]=0$ and $\left[v_{4} \circ \eta_{7}^{2}, \eta_{4}^{2}\right]=0$.

Next, $\left[\Sigma v^{\prime}, \iota_{4}\right]=\left[\iota_{4}, \iota_{4}\right] \circ \Sigma v^{\prime}$ and [20, (5.5), p. 42 and Proposition 5.11, p. 46] implies $\left[\Sigma v^{\prime}, \iota_{4}\right]= \pm 4 v_{2}^{2}$. Consequently,

$$
\left[\Sigma v^{\prime} \circ \eta_{7}^{2}, \iota_{4} \circ \eta_{4}^{2}\right]=\left[\Sigma v^{\prime}, \iota_{4}\right] \circ \Sigma\left(\eta_{6}^{2} \wedge \eta_{3}^{2}\right)=0
$$

This proves that $J\left(\eta_{4}, \eta_{4}^{2}, 2 \iota_{4}\right)=\left[\pi_{11}\left(\mathbb{S}^{4}\right), 2 \iota_{4}\right]$.

To determine its order, notice that by means of Serre's isomorphisms

$$
\pi_{13}\left(\mathbb{S}^{3} ; p\right) \oplus \pi_{14}\left(\mathbb{S}^{7} ; p\right) \simeq \pi_{14}\left(\mathbb{S}^{4} ; p\right)
$$

for $p=3,5$, the orders of $\left[\alpha_{2}(4), \iota_{4}\right]=\left[\iota_{4}, \iota_{4}\right] \circ \alpha_{2}(7)$ and $\left[\alpha_{1}(4), \iota_{4}\right]=\left[\iota_{4}, \iota_{4}\right] \circ \alpha_{1}(7)$ are three and five, respectively. This implies that the order of $J\left(\eta_{4}, \eta_{4}^{2}, 2 \iota_{4}\right)$ is fifteen.

Finally, let $\alpha \in \pi_{14}\left(\mathbb{S}^{4}\right)$ be such that $\left[\eta_{4}, \eta_{4}^{2}, 2 \iota_{4}\right]=\alpha+J\left(\eta_{4}, \eta_{4}^{2}, 2 \iota_{4}\right)$. Since $2 \eta_{4}=0$, in view of Corollaries 1 and 3 , we deduce that

$$
0 \in\left[2 \eta_{4}, \eta_{4}^{2}, 2 \iota_{4}\right] \subseteq 2\left[\eta_{4}, \eta_{4}^{2}, 2 \iota_{4}\right]=2 \alpha+J\left(\eta_{4}, \eta_{4}^{2}, 2 \iota_{4}\right)
$$

Consequently, $2 \alpha \in J\left(\eta_{4}, \eta_{4}^{2}, 2 \iota_{4}\right)$ and so $30 \alpha=0$. Hence, $\alpha=a \alpha^{\prime}+b \beta$ for $\alpha^{\prime} \in \pi_{14}^{4}$ with order two and $\beta \in J\left(\eta_{4}, \eta_{4}^{2}, 2 \iota_{4}\right)$ for some $a \in\{0,1\}$ and $b \in\{0, \ldots, 14\}$. This implies that $\left[\eta_{4}, \eta_{4}^{2}, 2 \iota_{4}\right]=\alpha+J\left(\eta_{4}, \eta_{4}^{2}, 2 \iota_{4}\right)=a \alpha^{\prime}+J\left(\eta_{4}, \eta_{4}^{2}, 2 \iota_{4}\right)$ and $a \alpha^{\prime} \in\left[\eta_{4}, \eta_{4}^{2}, 2 \iota_{4}\right]$.

In view of [20, Theorem 7.3, p. 66], it holds $\pi_{14}^{4}=\mathbb{Z}_{8}\left\{v_{4} \circ \sigma^{\prime}\right\} \oplus \mathbb{Z}_{4}\left\{\Sigma \varepsilon^{\prime}\right\} \oplus$ $\mathbb{Z}_{2}\left\{\eta_{4} \circ \mu_{5}\right\}$. Then, we derive that $\alpha^{\prime}=4 x^{\prime}\left(v_{4} \circ \sigma^{\prime}\right)+2 y^{\prime}\left(\Sigma \varepsilon^{\prime}\right)+z^{\prime}\left(\eta_{4} \circ \mu_{5}\right)$ for some $x^{\prime}, y^{\prime}, z^{\prime} \in\{0,1\}$. Because $\alpha^{\prime} \in\left[\eta_{4}, \eta_{4}^{2}, 2 \iota_{4}\right]$, we get from Theorem 2(iii) that $0=$ $\Sigma \alpha^{\prime}=4 x^{\prime}\left(\nu_{5} \circ \Sigma \sigma^{\prime}\right)+2 y^{\prime}\left(\Sigma^{2} \varepsilon^{\prime}\right)+z^{\prime}\left(\eta_{5} \circ \mu_{6}\right)$. But, by [20, (7.10), p. 67 and (7.16), p. 69], it holds $2\left(\nu_{5} \circ \sigma_{8}\right)= \pm \Sigma^{2} \varepsilon^{\prime}$ and $2\left(\nu_{5} \circ \sigma_{8}\right)=\nu_{5} \circ \Sigma \sigma^{\prime}$. Thus, $z^{\prime}\left(\eta_{5} \circ \mu_{6}\right)=0$, so $z^{\prime}=0$ and $\alpha^{\prime}=4 x^{\prime}\left(v_{4} \circ \sigma^{\prime}\right)+2 y^{\prime}\left(\Sigma \varepsilon^{\prime}\right)$ for some $x^{\prime}, y^{\prime} \in\{0,1\}$ which completes the proof.

\section{Main result}

Theorem 1 provides the following commutative diagram

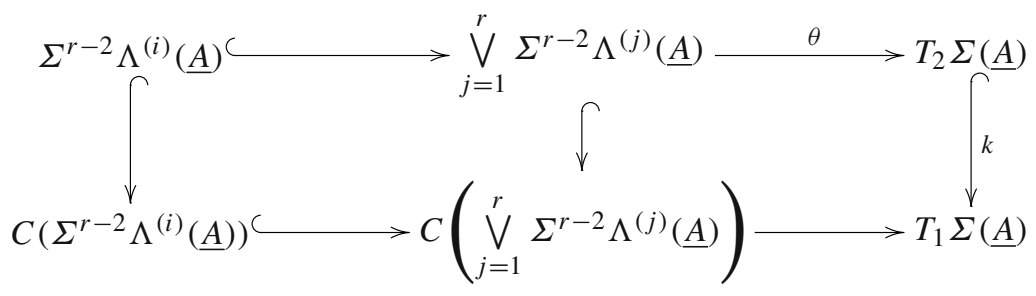


which lead to pair-maps

$$
\left(h_{i}^{\prime}, h_{i}^{\prime \prime}\right):\left(C\left(\Sigma^{r-2} \Lambda^{(i)}(\underline{A})\right), \Sigma^{r-2} \Lambda^{(i)}(\underline{A})\right) \rightarrow\left(T_{1} \Sigma(\underline{A}), T_{2} \Sigma(\underline{A})\right),
$$

for $i=1, \ldots, r$.

Let $\Theta_{i}=\left(h_{i}^{\prime}, h_{i}^{\prime \prime}\right) \in \pi_{1}\left(\Sigma^{r-2} \Lambda^{(i)}(\underline{A}), k\right)$ and $J_{i}: \Sigma A_{i} \hookrightarrow T_{2} \Sigma(\underline{A})$ be the canonical inclusion map for $i=1, \ldots, r$, where $k: T_{2} \Sigma(\underline{A}) \hookrightarrow T_{1} \Sigma(\underline{A})$ is the inclusion map. Then each relative generalized Whitehead product

$$
\begin{aligned}
{\left[\Theta_{i}, J_{i}\right]_{R}: } & \left(C\left(\Sigma\left(\Sigma^{r-3} \Lambda^{(i)}(\underline{A}) \wedge A_{i}\right)\right), \Sigma\left(\Sigma^{r-3} \Lambda^{(i)}(\underline{A}) \wedge A_{i}\right)\right) \\
& \rightarrow\left(T_{1} \Sigma(\underline{A}), T_{2} \Sigma(\underline{A})\right)
\end{aligned}
$$

simplifies to

$$
\tau_{i}^{*}\left[\Theta_{i}, J_{i}\right]_{R}:\left(C\left(\Sigma^{r-2} \Lambda(\underline{A})\right), \Sigma^{r-2} \Lambda(\underline{A})\right) \rightarrow\left(T_{1} \Sigma(\underline{A}), T_{2} \Sigma(\underline{A})\right)
$$

where $\tau_{i}=\left(C \Sigma^{r-2} \sigma_{i}, \Sigma^{r-2} \sigma_{i}\right)$ for $i=1, \ldots, r$, and $\sigma_{i} \in S_{r}$ is the permutation inducing the homeomorphism $\sigma_{i}: \Lambda(\underline{A}) \rightarrow \Lambda^{(i)}(\underline{A}) \wedge A_{i}$.

Hence, we got the relative generalized Whitehead products $\tau_{i}^{*}\left[\Theta_{i}, J_{i}\right]_{R} \in$ $\pi_{1}\left(\Sigma^{r-2} \Lambda(\underline{A}), k\right)$ for $i=1, \ldots, r$.

Next, consider the commutative diagram

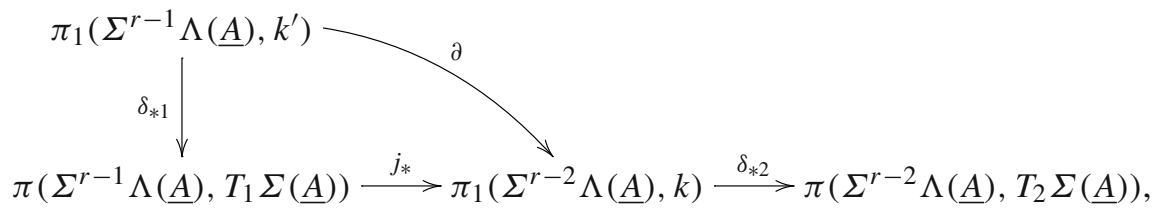

where $k: T_{2} \Sigma(\underline{A}) \hookrightarrow T_{1} \Sigma(\underline{A})$ and $k^{\prime}: T_{1} \Sigma(\underline{A}) \hookrightarrow T_{0} \Sigma(\underline{A})$ are inclusion maps, $\delta_{* 1}, \delta_{* 2}$ are boundaries and $j_{*}$ is the obvious map.

Since the bottom row is exact and $\delta_{* 1}\left(\Omega_{r}, \omega_{r}\right)=\omega_{r}$, we obtain $\partial\left(\Omega_{r}, \omega_{r}\right)=j_{*}\left(\omega_{r}\right)$ and so $\delta_{* 2} \partial\left(\Omega_{r}, \omega_{r}\right)=\delta_{* 2} j_{*}\left(\omega_{r}\right)=0$.

Following mutatis mutandis the result due to Nakaoka-Toda [14, Lemma (1.2)] for spheres and a proof of its generalization [10, Formula (0.1)], we may state for suspensions:

Lemma 3 If $h=\sum_{i=1}^{r} \tau_{i}^{*}\left[\Theta_{i}, J_{i}\right]_{R} \in \pi_{1}\left(\Sigma^{r-2} \Lambda(\underline{A}), k\right)$ then $h=\partial\left(\Omega_{r}, \omega_{r}\right)$.

Notice that in view of the formula (3), we have

$$
\left[\Theta_{i}, J_{i}\right]_{R}=\left(\left[h_{i}^{\prime}, k_{J_{i}}\right]^{\prime},\left[h_{i}^{\prime \prime}, J_{i}\right]\right)
$$

for $i=1, \ldots, r$. Consequently,

$$
h=\left(h^{\prime}, h^{\prime \prime}\right)=\left(\sum_{i=1}^{r}\left(C \Sigma^{r-2} \sigma_{i}\right)^{*}\left[h_{i}^{\prime}, k J_{i}\right]^{\prime}, \sum_{i=1}^{r}\left(\Sigma^{r-2} \sigma_{i}\right)^{*}\left[h_{i}^{\prime \prime}, J_{i}\right]\right) .
$$


Now, we are in a position to generalize the main result of [8, Theorem 4.3].

Theorem 4 Let $f: T_{2} \Sigma(\underline{A}) \rightarrow X$ be any map, $J_{i}: \Sigma A_{i} \hookrightarrow T_{2} \Sigma(\underline{A})$ be the canonical inclusion maps, and $f_{i}=f_{J_{i}}: \Sigma A_{i} \rightarrow X$ for $i=1, \ldots, r$.

(i) If $f^{\prime}, f^{\prime \prime}: T_{1} \Sigma(\underline{A}) \rightarrow X$ are extensions of $f$ then

$$
\omega_{r}\left(f^{\prime}\right)-\omega_{r}\left(f^{\prime \prime}\right) \in \sum_{i=1}^{r}\left(\Sigma^{r-1} \sigma_{i}\right)^{*}\left[\pi\left(\Sigma^{r-1} \Lambda^{(i)}(\underline{A}), X\right), f_{i}\right],
$$

where $\sigma_{i} \in S_{r}$ is the permutation inducing the homeomorphism $\sigma_{i}: \Lambda(\underline{A}) \rightarrow$ $\Lambda^{(i)}(\underline{A}) \wedge A_{i}$ for $i=1, \ldots, r$.

(ii) If $f^{\prime}: T_{1} \Sigma(\underline{A}) \rightarrow X$ is an extension of $f$ then for any

$$
\gamma \in \sum_{i=1}^{r}\left(\Sigma^{r-1} \sigma_{i}\right)^{*}\left[\pi\left(\Sigma^{r-1} \Lambda^{(i)}(\underline{A}), X\right), f_{i}\right]
$$

there exists $f^{\prime \prime}: T_{1} \Sigma(\underline{A}) \rightarrow X$ an extension of $f$ such that $\gamma=\omega_{r}\left(f^{\prime}\right)-\omega_{r}\left(f^{\prime \prime}\right)$.

Proof (i) By Lemma 3 and commutativity of the diagram above, $h=\partial\left(\Omega_{r}, \omega_{r}\right)=$ $j_{*}\left(\omega_{r}\right)$ so that $\omega_{r} \in j_{*}^{-1}(h)$. In view of Proposition 1(viii), there exists $g^{\prime}$ : $C \Sigma^{r-2} \Lambda(\underline{A}) \rightarrow T_{2} \Sigma(\underline{A})$ with $g_{\mid \Sigma^{r-2} \Lambda(\underline{A})}^{\prime}=h_{\mid \Sigma^{r-2} \Lambda(\underline{A})}^{\prime}=h^{\prime \prime}$ such that $\omega_{r}=$ $d\left(h^{\prime}, k g^{\prime}\right) \in \pi\left(\Sigma^{r-1} \Lambda(\underline{A}), T_{1} \Sigma(\underline{A})\right)$.

Since $f^{\prime} k=f^{\prime \prime} k=f$, by (4) and Propositions 1-2, we get

$$
\begin{aligned}
& \omega_{r}\left(f^{\prime}\right)-\omega_{r}\left(f^{\prime \prime}\right)=d\left(f^{\prime} h^{\prime}, f^{\prime} k g^{\prime}\right)-d\left(f^{\prime \prime} h^{\prime}, f^{\prime \prime} k g^{\prime}\right) \\
& =d\left(f^{\prime} h^{\prime}, f^{\prime \prime} h^{\prime}\right) \\
& =d\left(\sum_{i=1}^{r} f^{\prime}\left(\left(C \Sigma^{r-2} \sigma_{i}\right)^{*}\left[h_{i}^{\prime}, k J_{i}\right]^{\prime}\right), \sum_{i=1}^{r} f^{\prime \prime}\left(\left(C \Sigma^{r-2} \sigma_{i}\right)^{*}\left[h_{i}^{\prime}, k J_{i}\right]^{\prime}\right)\right) \\
& =\sum_{i=1}^{r} d\left(\left(C \Sigma^{r-2} \sigma_{i}\right)^{*}\left[f^{\prime} h_{i}^{\prime}, f^{\prime} k_{J i}\right]^{\prime},\left(C \Sigma^{r-2} \sigma_{i}\right)^{*}\left[f^{\prime \prime} h_{i}^{\prime}, f^{\prime \prime} k_{J_{i}}\right]^{\prime}\right) \\
& =\sum_{i=1}^{r}\left(\Sigma^{r-1} \sigma_{i}\right)^{*}\left[d\left(f^{\prime} h_{i}^{\prime}, f^{\prime \prime} h_{i}^{\prime}\right), f_{i}\right] \\
& \in \sum_{i=1}^{r}\left(\Sigma^{r-1} \sigma_{i}\right)^{*}\left[\pi\left(\Sigma^{r-1} \Lambda^{(i)}(\underline{A}), X\right), f_{i}\right] .
\end{aligned}
$$

(ii) Let $\gamma=\sum_{i=1}^{r}\left(\Sigma^{r-1} \sigma_{i}\right)^{*}\left[\gamma_{i}, f_{i}\right]$, with $\gamma_{i}: \Sigma^{r-1} \Lambda^{(i)}(\underline{A}) \rightarrow X$. By (7), we obtain $f^{\prime} h_{i}^{\prime}: C\left(\Sigma^{r-2} \Lambda^{(i)}(\underline{A})\right) \rightarrow X$ and then, in view of Proposition 1(vii), there exist maps $g_{i}: C\left(\Sigma^{r-2} \Lambda^{(i)}(\underline{A})\right) \rightarrow X$ such that the restrictions $g_{i \mid \Sigma^{r-2} \Lambda^{(i)}(\underline{A})}=$ $f h_{i \mid \Sigma^{r-2} \Lambda^{(i)}(\underline{A})}^{\prime \prime}$ and $d\left(f^{\prime} h_{i}^{\prime}, g_{i}\right)=\gamma_{i}$ for $i=1, \ldots, r$.

Note that the maps $g_{i}$ determine a map

$$
g=g_{1} \vee \cdots \vee g_{r}: C\left(\bigvee_{j=1}^{r} \Sigma^{r-2} \Lambda^{(j)}(\underline{A})\right) \rightarrow X .
$$


Since, by Theorem 1 , the space $T_{1} \Sigma(\underline{A})$ is a push-out, the universal property guarantees the existence of $f^{\prime \prime}: T_{1} \Sigma(\underline{A}) \rightarrow X$ with $f_{\mid T_{2} \Sigma(\underline{A})}^{\prime \prime}=f_{\mid T_{2} \Sigma(\underline{A})}^{\prime}=f$ and $f^{\prime \prime} h_{i}^{\prime}=g_{i}$.

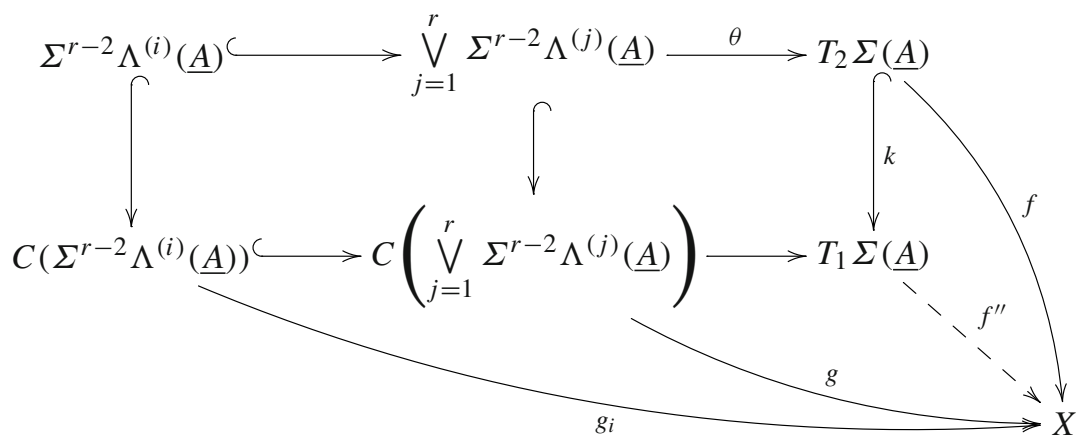

The same computations as in (i) show that $\gamma=\omega_{r}\left(f^{\prime}\right)-\omega_{r}\left(f^{\prime \prime}\right)$ and the proof is complete.

Corollary 4 (i) Let $f_{i}: \Sigma A_{i} \rightarrow X$ be any maps for $i=1, \ldots, r$. Suppose that $\left[f_{1}, \ldots, f_{r}\right] \neq \emptyset$. Then for any $\beta \in\left[f_{1}, \ldots, f_{r}\right]$ there is an inclusion

$$
\beta+\sum_{i=1}^{r}\left(\Sigma^{r-1} \sigma_{i}\right)^{*}\left[\pi\left(\Sigma^{r-1} \Lambda^{(i)}(\underline{A}), X\right), f_{i}\right] \subseteq\left[f_{1}, \ldots, f_{r}\right]
$$

(ii) If $\omega_{r}(f) \in\left[f_{1}, \ldots, f_{r}\right]$ for $f: T_{1} \Sigma(\underline{A}) \rightarrow X$ then

$$
\begin{aligned}
\sum_{i=1}^{r}\left(\Sigma^{r-1} \sigma_{i}\right)^{*}\left[\pi\left(\Sigma^{r-1} \Lambda^{(i)}(\underline{A}), X\right), f_{i}\right] & = \\
\left\{\omega_{r}\left(f^{\prime}\right)-\omega_{r}(f) ; f^{\prime}: T_{1} \Sigma(\underline{A})\right. & \rightarrow X \text { with } f_{\mid T_{2} \Sigma(\underline{A})}^{\prime}=f_{\left.\mid T_{2} \Sigma(\underline{A})\right\} .}
\end{aligned}
$$

(iii) If $\left[f_{1}, f_{2}, f_{3}\right] \neq \varnothing$ then it is a coset of the subgroup

$$
\begin{gathered}
\left(\Sigma^{2} \sigma_{1}\right)^{*}\left[\pi\left(\Sigma^{2} \Lambda^{(1)}(\underline{A}), X\right), f_{1}\right]+\left(\Sigma^{2} \sigma_{2}\right)^{*}\left[\pi\left(\Sigma^{2} \Lambda^{(2)}(\underline{A}), X\right), f_{2}\right] \\
+\left(\Sigma^{2} \sigma_{3}\right)^{*}\left[\pi\left(\Sigma^{2} \Lambda^{(3)}(\underline{A}), X\right), f_{3}\right] \subseteq \pi\left(\Sigma^{2} \Lambda(\underline{A}), X\right) .
\end{gathered}
$$

Proof (i) First, for simplicity, let $\Gamma=\sum_{i=1}^{r}\left(\Sigma^{r-1} \sigma_{i}\right)^{*}\left[\pi\left(\Sigma^{r-1} \Lambda^{(i)}(\underline{A}), X\right), f_{i}\right]$. Next, let $f^{\prime}: T_{1} \Sigma(\underline{A}) \rightarrow X$ be a map such that $\beta=\omega_{r}\left(f^{\prime}\right)$. Let $f=f_{\mid T_{2} \Sigma(\underline{A})}^{\prime}$ : $T_{2} \Sigma(\underline{A}) \rightarrow X$ so that $f^{\prime}$ extends $f$, obviously. By Theorem 4(ii), for any $\gamma \in \Gamma$ there exists $f^{\prime \prime}: T_{1} \Sigma(\underline{A}) \rightarrow X$ an extension of $f$ such that $\gamma=\omega_{r}\left(f^{\prime}\right)-\omega_{r}\left(f^{\prime \prime}\right)$, or equivalently, $\beta+(-\gamma)=\omega_{r}\left(f^{\prime \prime}\right)$. Finally, since $f^{\prime \prime}$ extends the wedge sum map $f_{1} \vee \cdots \vee f_{r}$, then $\omega_{r}\left(f^{\prime \prime}\right) \in\left[f_{1}, \ldots, f_{r}\right]$ and the result follows.

(ii) This follows directly from Theorem 4.

(iii) This is a consequence of (i) and (ii). 


\section{Some computations for spheres and projective spaces}

Because $0 \in\left[0_{1}, 0_{2}, 0_{3}\right]$, Corollary 4(iii) implies $\left[0_{1}, 0_{2}, 0_{3}\right]=0$ (modulo indeterminacy). Further, we can easily show:

Lemma 4 Let $0_{i}: \Sigma A_{i} \rightarrow X$ be trivial maps for $i=1, \ldots, r$ with $r \geq 3$. The following are equivalent:

(i) $\left[0_{1}, \ldots, 0_{r}\right]=0$ (modulo indeterminacy) for any space $X$;

(ii) the composition

$$
\Sigma^{r-1} \Lambda(\underline{A}) \stackrel{\omega_{r}}{\rightarrow} T_{1} \Sigma(\underline{A}) \stackrel{p}{\rightarrow} T_{1} \Sigma(\underline{A}) / T_{r-1} \Sigma(\underline{A})
$$

is trivial, where $p$ is the quotient map;

(iii) there is a retraction

$$
T_{0} \Sigma(\underline{A}) / T_{r-1} \Sigma(\underline{A}) \stackrel{\rho}{\rightarrow} T_{1} \Sigma(\underline{A}) / T_{r-1} \Sigma(\underline{A})
$$

for the map

$$
T_{1} \Sigma(\underline{A}) / T_{r-1} \Sigma(\underline{A}) \stackrel{\bar{k}}{\rightarrow} T_{0} \Sigma(\underline{A}) / T_{r-1} \Sigma(\underline{A})
$$

induced by the inclusion $k: T_{1} \Sigma(\underline{A}) \hookrightarrow T_{0} \Sigma(\underline{A})$.

Proposition 5 If $r \geq 4$ and $\underline{\mathbb{S}}=\left(\mathbb{S}^{m_{1}-1}, \ldots, \mathbb{S}^{m_{r}-1}\right)$ with $m_{i} \geq 1$ for $i=1, \ldots, r$ then the quotient map $T_{1} \Sigma(\underline{\mathbb{S}}) \rightarrow T_{1} \Sigma(\underline{\mathbb{S}}) / T_{r-1} \Sigma(\underline{\mathbb{S}})$ leads to a non-trivial element of the rth order generalized Whitehead product $\left[0_{1}, \ldots, 0_{r}\right]$.

Proof ${ }^{1}$ Suppose that the quotient map $T_{1} \Sigma(\underline{\mathbb{S}}) \rightarrow T_{1} \Sigma(\underline{\mathbb{S}}) / T_{r-1} \Sigma(\underline{\mathbb{S}})$ yields a trivial element of the $r$ th order generalized Whitehead product $\left[0_{1}, \ldots, 0_{r}\right]$ for $r \geq 4$. Then, by Lemma 4(iii), there is a retraction

$$
T_{0} \Sigma(\underline{\mathbb{S}}) / T_{r-1} \Sigma(\underline{\mathbb{S}}) \stackrel{\rho}{\rightarrow} T_{1} \Sigma(\underline{\mathbb{S}}) / T_{r-1} \Sigma(\underline{\mathbb{S}})
$$

for the map

$$
T_{1} \Sigma(\underline{\mathbb{S}}) / T_{r-1} \Sigma(\underline{\mathbb{S}}) \stackrel{\bar{k}}{\rightarrow} T_{0} \Sigma(\underline{A}) / T_{r-1} \Sigma(\underline{A})
$$

induced by the inclusion $k: T_{1} \Sigma(\underline{\mathbb{S}}) \hookrightarrow T_{0} \Sigma(\underline{\mathbb{S}})$.

This leads to homomorphisms of cohomology algebras

$$
\rho^{*}: H^{*}\left(T_{1} \Sigma(\underline{\mathbb{S}}) / T_{r-1} \Sigma(\underline{\mathbb{S}})\right) \rightarrow H^{*}\left(T_{0} \Sigma(\underline{\mathbb{S}}) / T_{r-1} \Sigma(\underline{\mathbb{S}})\right)
$$

\footnotetext{
1 We are deeply grateful to Jie Wu for his idea on the presented proof.
} 
and

$$
\bar{k}^{*}: H^{*}\left(T_{0} \Sigma(\underline{\mathbb{S}}) / T_{r-1} \Sigma(\underline{\mathbb{S}})\right) \rightarrow H^{*}\left(T_{1} \Sigma(\underline{\mathbb{S}}) / T_{r-1} \Sigma(\underline{\mathbb{S}})\right)
$$

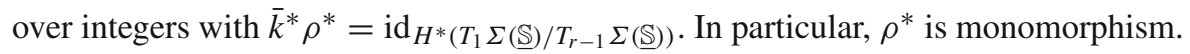
The long exact sequence for the triple $T_{r-1} \Sigma(\underline{\mathbb{S}}) \subseteq T_{1} \Sigma(\underline{\mathbb{S}}) \subseteq T_{0} \Sigma(\underline{\mathbb{S}})$ yields homomorphisms

$$
\bar{k}^{*}: H^{n}\left(T_{0} \Sigma(\underline{\mathbb{S}}) / T_{r-1} \Sigma(\underline{\mathbb{S}})\right) \rightarrow H^{n}\left(T_{1} \Sigma(\underline{\mathbb{S}}) / T_{r-1} \Sigma(\underline{\mathbb{S}})\right)
$$

which is an isomorphism for $n<m_{1}+\cdots+m_{r}-1$, and a monomorphism for $n<m_{1}+\cdots+m_{r}$.

Let now $i: T_{r-1} \Sigma(\underline{\mathbb{S}}) \hookrightarrow T_{0} \Sigma(\underline{\mathbb{S}})$ and $q: T_{0} \Sigma(\underline{\mathbb{S}}) \rightarrow T_{0} \Sigma(\underline{\mathbb{S}}) / T_{r-1} \Sigma(\underline{\mathbb{S}})$ be the inclusion and quotient maps, respectively. Thus, the Künneth Theorem implies that the induced cohomology maps $i^{*}: H^{n}\left(T_{0} \Sigma(\underline{\mathbb{S}})\right) \rightarrow H^{n}\left(T_{r-1} \Sigma(\underline{\mathbb{S}})\right)$ are splitting epimorphisms for $n \geq 0$, and consequently

$$
q^{*}: H^{*}\left(T_{0} \Sigma(\underline{\mathbb{S}}) / T_{r-1} \Sigma(\underline{\mathbb{S}})\right) \rightarrow H^{*}\left(T_{0} \Sigma(\underline{\mathbb{S}})\right)
$$

is a monomorphism of cohomology algebras.

Now, consider the non-trivial elements $\alpha \in H^{m_{1}+m_{2}}\left(T_{1} \Sigma(\underline{\mathbb{S}}) / T_{r-1} \Sigma(\underline{\mathbb{S}})\right)$ and $\beta \in H^{m_{3}+\cdots+m_{r}}\left(T_{1} \Sigma(\underline{\mathbb{S}}) / T_{r-1} \Sigma(\underline{\mathbb{S}})\right)$ determined by cocycles associated to the cells $e^{m_{1}} \times e^{m_{2}}$ and $e^{m_{3}} \times \cdots \times e^{m_{r}}$, respectively. Then, the cup-product $\alpha \smile \beta \in$ $H^{m_{1}+\cdots+m_{r}}\left(T_{1} \Sigma(\underline{\mathbb{S}}) / T_{r-1} \Sigma(\underline{\mathbb{S}})\right)=0$ and consequently, $\rho^{*}(\alpha \smile \beta)=\rho^{*}(\alpha) \smile$ $\rho^{*}(\beta)=0$.

On the other hand, since $\rho^{*}$ and $q^{*}$ are monomorphisms, the elements $q^{*} \rho^{*}(\alpha)$ and $q^{*} \rho^{*}(\beta)$ are non-trivial as well. Further, in view of (8), the map

$$
\bar{k}^{*}: H^{n}\left(T_{0} \Sigma(\underline{\mathbb{S}}) / T_{r-1} \Sigma(\underline{\mathbb{S}})\right) \stackrel{\cong}{\rightarrow} H^{n}\left(T_{1} \Sigma(\underline{\mathbb{S}}) / T_{r-1} \Sigma(\underline{\mathbb{S}})\right)
$$

is an isomorphism with the inverse

$$
\rho^{*}=\left(\bar{k}^{*}\right)^{-1}: H^{n}\left(T_{1} \Sigma(\underline{\mathbb{S}}) / T_{r-1} \Sigma(\underline{\mathbb{S}})\right) \stackrel{\cong}{\rightarrow} H^{n}\left(T_{0} \Sigma(\underline{\mathbb{S}}) / T_{r-1} \Sigma(\underline{\mathbb{S}})\right)
$$

for $n=m_{1}+m_{2}$ and $n=m_{3}+\cdots+m_{r}$.

Then, the cohomology algebra structure

$$
H^{*}\left(T_{0} \Sigma(\underline{\mathbb{S}})\right) \stackrel{\cong}{\rightarrow} H^{*}\left(\mathbb{S}^{m_{1}}\right) \otimes \cdots \otimes H^{*}\left(\mathbb{S}^{m_{r}}\right)
$$

and $r \geq 4$ lead to the non-trivial cup-product

$$
q^{*} \rho^{*}(\alpha) \smile q^{*} \rho^{*}(\beta)=q^{*}\left(\rho^{*}(\alpha) \smile \rho^{*}(\beta)\right) \in H^{m_{1}+\cdots+m_{r}}\left(T_{0} \Sigma(\underline{\mathbb{S}})\right) .
$$

Consequently, $\rho^{*}(\alpha) \smile \rho^{*}(\beta) \in H^{m_{1}+\cdots+m_{r}}\left(T_{0} \Sigma(\underline{\mathbb{S}}) / T_{r-1} \Sigma(\underline{\mathbb{S}})\right)$ is non-trivial as well. This contradiction completes the proof. 
Remark 4 The generalized Whitehead map $\omega_{r}: \Sigma^{r-1} \Lambda(\underline{\mathbb{S}}) \rightarrow T_{1} \Sigma(\underline{\mathbb{S}})$ is non-trivial for any $r \geq 2$. Indeed, if $\omega_{r}$ is trivial then it factors through the cone $C \Sigma^{r-1} \Lambda(\underline{\mathbb{S}})$. Hence, in view of (6), there exists a retraction $\rho: T_{0} \Sigma(\underline{\mathbb{S}}) \rightarrow T_{1} \Sigma(\underline{\mathbb{S}})$ for the inclusion $\operatorname{map} k: T_{1} \Sigma(\underline{\mathbb{S}}) \hookrightarrow T_{0} \Sigma(\underline{\mathbb{S}})$.

Let $\alpha \in H^{m_{1}}\left(T_{1} \Sigma(\underline{\mathbb{S}})\right)$ and $\beta \in H^{m_{2}+\cdots+m_{r}}\left(T_{1} \Sigma(\underline{\mathbb{S}})\right)$ be non-trivial elements determined by cocycles associated to the cells $e^{m_{1}}$ and $e^{m_{2}} \times \cdots \times e^{m_{r}}$, respectively. Thus,

$$
\alpha \smile \beta \in H^{m_{1}+\cdots+m_{r}}\left(T_{1} \Sigma(\underline{\mathbb{S}})\right)=0 .
$$

But, $\rho^{*}(\alpha), \rho^{*}(\beta) \in H^{*}\left(T_{0} \Sigma(\underline{\mathbb{S}})\right)$ satisfy

$$
\rho^{*}(\alpha) \smile \rho^{*}(\beta)=\rho^{*}(\alpha \smile \beta) \neq 0
$$

and since $\rho^{*}$ is monomorphism, it holds $\alpha \smile \beta \neq 0$. This contradiction guarantees that $\omega_{r}$ is non-trivial.

Let $\mathbb{R}$ and $\mathbb{C}$ be the fields of real and complex numbers, respectively and $\mathbb{H}$ the skew $\mathbb{R}$-algebra of quaternions. Denote by $\mathbb{F} P^{n}$ the $n$-projective space over $\mathbb{F}=\mathbb{R}$, $\mathbb{C}$ or $\mathbb{H}$, put $d=\operatorname{dim}_{\mathbb{R}} \mathbb{F}$ and set $i_{n \mathbb{F}}: \mathbb{S}^{d} \hookrightarrow \mathbb{F} P^{n}$ for the inclusion map. Let $\gamma_{n \mathbb{F}}: \mathbb{S}^{(n+1) d-1} \rightarrow \mathbb{F} P^{n}$ be the canonical quotient map. In view of [3, Corollary (7.4)] and $[4,(4.1-3)]$, we obtain a key formula:

Lemma 5 Let $h_{0} f \in \pi_{k}\left(\mathbb{S}^{2 n-1}\right)$ be the 0th Hopf-Hilton invariant for $f \in \pi_{k}\left(\mathbb{S}^{n}\right)$. Then:

$$
\left[\gamma_{n \mathbb{R}} f, i_{n \mathbb{R}}\right]= \begin{cases}0, & \text { for odd } n \\ (-1)^{k} \gamma_{n \mathbb{R}}\left(-2 f+\left[\iota_{n}, \iota_{n}\right] \circ h_{0} f\right), & \text { for even } n\end{cases}
$$

Now, let $f: T_{1} \Sigma(\underline{\mathbb{S}}) \rightarrow \mathbb{S}^{2}$ for $\underline{\mathbb{S}}=\left(\mathbb{S}^{m_{1}-1}, \ldots, \mathbb{S}^{m_{r}-1}\right)$. Recall that by [5, Satz], we have $\omega_{r}(f)=0$ provided $m_{1}+\cdots+m_{r} \neq 4$. Further, Lemma 5 implies that $\left[\pi_{r-1}\left(\mathbb{R} P^{2}\right), i_{2 \mathbb{R}}\right] \neq 0$ in general. This yields the following result.

Proposition 6 Let $f: T_{1} \Sigma(\underline{\mathbb{S}}) \rightarrow \mathbb{R} P^{2}$ for $\underline{\mathbb{S}}=\left(\mathbb{S}^{m_{1}-1}, \ldots, \mathbb{S}^{m_{r}-1}\right)$.

(i) If $m_{i} \geq 2$ for $i=1, \ldots, r$ with $r \geq 2$, then $\omega_{r}(f)=0$ provided $m_{1}+\cdots+m_{r} \neq$ 4.

(ii) $\left[\pi_{r-1}\left(\mathbb{R} P^{2}\right), i_{2} \mathbb{R}\right] \subseteq\left[f_{1}, \ldots, f_{r}\right]$ for $f_{i}: \mathbb{S}^{1} \rightarrow \mathbb{R} P^{2}$ with $i=1, \ldots, r$ and $f_{i_{0}}=i_{2 \mathbb{R}}$ for at least one $1 \leq i_{0} \leq r$. In particular, $2 \pi_{2}\left(\mathbb{R} P^{2}\right)=\left[0,0, i_{2 \mathbb{R}}\right]=$ $\left[0, i_{2 \mathbb{R}}, i_{2 \mathbb{R}}\right]=\left[i_{2 \mathbb{R}}, i_{2 \mathbb{R}}, i_{2 \mathbb{R}}\right]$

Proof (i) Since $m_{i} \geq 2$ for $i=1, \ldots, r$, the space $T_{1} \Sigma(\mathbb{S})$ is 1 -connected. Hence, any map $f: T_{1} \Sigma(\underline{\mathbb{S}}) \rightarrow \mathbb{R} P^{2}$ lifts to $\widetilde{f}: T_{1} \Sigma(\underline{\mathbb{S}}) \rightarrow \mathbb{S}^{2}$ via the quotient map $\gamma_{2 \mathbb{R}}: \mathbb{S}^{2} \rightarrow \mathbb{R} P^{2}$. Consequently, in view of [5, Satz], $\omega_{r}(f)=0$.

(ii) Certainly, in view of Corollary 1 , it holds $0 \in\left[f_{1}, \ldots, f_{r}\right]$ if at least $f_{i_{0}}=0$ for some $1 \leq i_{0} \leq r$. Further, because the circle $\mathbb{S}^{1}$ is a topological group, we have $\left[\iota_{1}, . \times r ., \iota_{1}\right]=0$. Hence, by Theorem $2(\mathrm{i}), 0=\left(i_{2 \mathbb{R}}\right)_{*}\left[\iota_{1}, . \times r ., \iota_{1}\right] \in$ 
$\left[i_{2 \mathbb{R}}, . \times r, i_{2 \mathbb{R}}\right]$. Then, Corollary 4 leads to the inclusion $\left[\pi_{r-1}\left(\mathbb{R} P^{2}\right), i_{2 \mathbb{R}}\right] \subseteq$ $\left[f_{1}, \ldots, f_{r}\right]$ if $f_{i_{0}}=i_{2 \mathbb{R}}$ for at least one $1 \leq i_{0} \leq r$.

Finally, Lemma 5 and $[8$, Theorem 0.1$]$ lead to $\left[\pi_{2}\left(\mathbb{R} P^{2}\right), i_{2 \mathbb{R}}\right]=2 \pi_{2}\left(\mathbb{R} P^{2}\right)=$ $\left[0,0, i_{2 \mathbb{R}}\right]=\left[0, i_{2 \mathbb{R}}, i_{2 \mathbb{R}}\right]=\left[i_{2 \mathbb{R}}, i_{2 \mathbb{R}}, i_{2 \mathbb{R}}\right]$ and the proof is complete.

Notice that we can also state:

Remark 5 (i) $0 \in\left[i_{n \mathbb{R}}, . \times r ., i_{n \mathbb{R}}\right] \subseteq \pi_{r-1}\left(\mathbb{R} P^{n}\right)$ for $r \geq 2$. In particular, $\left[i_{n \mathbb{R}}, . \times r, i_{n \mathbb{R}}\right]=0$ for $r \leq n$ and $2 \pi_{r-1}\left(\mathbb{R} P^{2 n}\right) \subseteq\left[i_{2 n \mathbb{R}}, . . \times r, i_{2 n \mathbb{R}}\right] \subseteq$ $\pi_{r-1}\left(\mathbb{R} P^{2 n}\right)$ for $r<4 n$.

(ii) $\left[i_{r} \mathbb{C}, \times(r+1), i_{r} \mathbb{C}\right]=(r+1) ! \gamma_{r} \mathbb{C}$ for $r \geq 2([16$, Corollary 2$])$.

(iii) It is known that $\left[i_{1 \mathbb{H}}, i_{1 \mathbb{H}}\right]=\left[\iota_{4}, \iota_{4}\right]= \pm\left(2 v_{4}-\Sigma \nu^{\prime}\right)$ and $\left[i_{n \mathbb{H}}, i_{n \mathbb{H}}\right]=$ $\left(i_{n \mathbb{H}}\right)_{*}\left[\iota_{4}, \iota_{4}\right]= \pm i_{n \mathbb{H}}\left(\Sigma \nu^{\prime}\right) \neq 0$ for $n \geq 2$. Hence, $\left[i_{n \mathbb{H}}, . \times r ., i_{n \mathbb{H}}\right]=\emptyset$ for $r \geq 3$ and $n \geq 1$.

Acknowledgements The authors are deeply grateful to the referee for a number of invaluable suggestions to work on the final version, especially on Proposition 4. They would like to thank Martin Arkowitz for a very helpful discussion and indicating the reference [1] as well.

Open Access This article is distributed under the terms of the Creative Commons Attribution 4.0 International License (http://creativecommons.org/licenses/by/4.0/), which permits unrestricted use, distribution, and reproduction in any medium, provided you give appropriate credit to the original author(s) and the source, provide a link to the Creative Commons license, and indicate if changes were made.

\section{References}

1. Ando, H.: On the generalized Whitehead products and the generalized Hopf invariant of a composition element. Tôhoku Math. J. 20(2), 516-553 (1968)

2. Arkowitz, M.: The generalized Whitehead product. Pac. J. Math. 12, 7-23 (1962)

3. Barcus, W.D., Barratt, M.G.: On the homotopy classification of the extensions of a fixed map. Trans. Am. Math. Soc. 88, 57-74 (1958)

4. Barratt, M.G., James, M., Stein, N.: Whitehead products and projective spaces. J. Math. Mech. 9, 813-819 (1960)

5. Baues, H.J.: Höhere Whitehead Produkte der zwei dimensionalen Sphäre. Comment. Math. Helv. 48, 116-118 (1973)

6. Davis, M.W., Januszkiewicz, T.: Convex polytopes, coxeter orbifolds and torus actions. Duke Math. J. 62, 417-451 (1991)

7. Grbić, J., Theriault, S.: Higher Whitehead products in toric topology. arXiv:1011.2133v2 [math.AT]

8. Hardie, K.A.: On a construction of E. C. Zeeman. J. Lond. Math. Soc. 35, 452-464 (1960)

9. Hardie, K.A.: Higher Whitehead products. Quart. J. Math. Oxf. Ser. 12(2), 241-249 (1961)

10. Hardie, K.A.: A proof of the Nakaoka-Toda formula. Pac. J. Math. 14, 1249-1254 (1964)

11. Iriye, K., Kishimoto, D.: Polyhedral products for shifted complexes and higher Whitehead products. arXiv:1505.04892 [math.AT]

12. James, I.M.: On spaces with a multiplication. Pac. J. Math. 7, 1083-1100 (1957)

13. Massey, W.S.: Some problems in algebraic topology and the theory of fibre bundles. Ann. Math. 62, 327-359 (1955)

14. Nakaoka, M., Toda, H.: On Jacobi identity for Whitehead products. J. Inst. Polytech. Osaka City Univ. Ser. A. 5(1), 1-13 (1954)

15. Porter, G.J.: Higher order Whitehead products. Topology 3, 123-135 (1965)

16. Porter, G.J.: Higher order Whitehead products and Postnikov systems. Ill. J. Math. 11, 414-416 (1967)

17. Salvatore, P.: Homotopy type of Euclidean configuration spaces. Rend. Circ. Mat. Palermo 66(Suppl. 2), 161-164 (2001)

18. Serre, J.-P.: Groupes d'homotopie et classes de groupes abéliens. Ann. Math. 58(2), 258-294 (1953)

19. Siegel, J.: $G$-spaces, $H$-spaces and $W$-spaces. Pac. J. Math. 31(1), 209-214 (1969) 
20. Toda, H.: Composition Methods in Homotopy Groups of Spheres. Annals of mathematics studies, issue 49. Princeton University Press, Princeton (1962)

21. Tsuchida, K.: Generalized James product and the Hopf construction. Tôhoku Math. J. 17(2), 319-334 (1965)

22. Williams, F.D.: A characterization of spaces with vanishing generalized higher Whitehead products. Bull. Am. Math. Soc. 74, 497-499 (1968) 